\title{
Frequent of ribosomal protein S6 hyperphosphorylation in lymphangioleiomyomatosis-associated angiomyolipomas
}

\author{
Victoria A Robb, Aristotelis Astrinidis and Elizabeth P Henske \\ Fox Chase Cancer Center, Philadelphia, PA, USA
}

\begin{abstract}
Lymphangioleiomyomatosis is a progressive lung disease characterized by a diffuse proliferation of pulmonary smooth muscle cells and cystic degeneration. Lymphangioleiomyomatosis can occur either independently of other disease or in association with tuberous sclerosis complex, a tumor-suppressor gene syndrome caused by mutations that inactivate either TSC1 or TSC2. TSC2 mutations and loss of heterozygosity have been identified in sporadic lymphangioleiomyomatosis-associated angiomyolipomas, thus implicating the TSC/Ras homologenriched in brain (Rheb)/mammalian target of Rapamycin (mTOR)/p70 S6 kinase signaling pathway in their pathogenesis. This study was undertaken to determine whether the mTOR/p70 S6 kinase signaling pathway is activated in lymphangioleiomyomatosis-associated angiomyolipomas lacking TSC1/TSC2 loss of heterozygosity. Phospho-ribosomal protein S6 (Ser235/236) immunohistochemistry was performed on five lymphangioleiomyomatosis-associated angiomyolipomas, two matched lymphangioleiomyomatosis pulmonary samples, and three sporadic angiomyolipomas. TSC1/TSC2 loss of heterozygosity was previously excluded in these angiomyolipomas. Moderate or strong phospho-ribosomal protein S6 immunoreactivity was found in all lymphangioleiomyomatosis-associated and sporadic angiomyolipomas, suggesting a high incidence of mTOR/p70 S6 kinase signaling pathway activation despite a lack of TSC1/TSC2 loss of heterozygosity. Focally positive phospho-S6 staining was also evident in both lymphangioleiomyomatosis pulmonary samples. We hypothesized that this $\mathbf{S 6}$ hyperphosphorylation could reflect mutational activation of Rheb or Rheb-like protein (RhebL1), Ras family members which directly activate mTOR. Mutational analysis performed on DNA from these eight angiomyolipomas plus five additional sporadic angiomyolipomas did not reveal mutations in exons 3 and 4 (homologous sites of Ras activating mutations) of either Rheb or RhebL1. These data suggest that activation of the Rheb/mTOR/p70 S6 kinase pathway is related to the pathogenesis of lymphangioleiomyomatosis-associated and sporadic angiomyolipomas lacking TSC1/TSC2 loss of heterozygosity. This high incidence of mTOR signaling pathway activation suggests that treatment with mTOR inhibitors, such as Rapamycin, may benefit patients with angiomyolipomas independent of the detection of TSC1/TSC2 loss of heterozygosity.
\end{abstract}

Modern Pathology (2006) 19, 839-846. doi:10.1038/modpathol.3800610; published online 31 March 2006

Keywords: angiomyolipomas; lymphangioleiomyomatosis; tuberous sclerosis complex; Rheb; mTOR; p70 S6 kinase

Lymphangioleiomyomatosis, a progressive lung disease affecting almost exclusively women, is characterized by a diffuse proliferation of smooth muscle cells and cystic degeneration. Lymphangioleiomyomatosis can occur either independently of other disease (sporadic) or in association with tuberous sclerosis complex. Tuberous sclerosis complex is a

Correspondence: Dr EP Henske, MD, Fox Chase Cancer Center, 333 Cottman Avenue, Philadelphia, PA 19111, USA.

E-mail: Elizabeth.Henske@fccc.edu

Received 26 January 2006; revised 15 March 2006; accepted 16 March 2006; published online 31 March 2006 tumor-suppressor gene syndrome caused by mutations that inactivate either TSC1 (the gene encoding the protein hamartin) or TSC2 (the gene encoding the protein tuberin) and is characterized by seizures, mental retardation, autism, and benign tumors of the brain, retina, kidney, heart, and skin. In a Mayo Clinic study of patients with tuberous sclerosis complex, lymphangioleiomyomatosis was the third most frequent cause of death after renal disease and brain tumors. ${ }^{1}$ Renal angiomyolipomas, benign tumors of the kidney composed of three distinct components: dysplastic blood vessels, smooth muscle cells, and adipose cells, ${ }^{2}$ can arise sporadically 
or in association with tuberous sclerosis complex or lymphangioleiomyomatosis. Angiomyolipomas occur in up to $80 \%$ of patients with tuberous sclerosis complex, and in up to $60 \%$ of patients with lymphangioleiomyomatosis.

TSC1 and TSC2 are classic tumor-suppressor genes, fitting the 'two hit' tumor-suppressor gene model. In this model, a germline mutation inactivates one allele and a somatic mutation or somatic chromosomal deletion (referred to as loss of heterozygosity) inactivates the remaining allele. Loss of heterozygosity in the TSC1 or TSC2 region occurs in most angiomyolipomas, rhabdomyomas, and astrocytomas from tuberous sclerosis complex patients. $^{3}$ TSC2 loss of heterozygosity also occurs in $10 \%$ of sporadic angiomyolipomas ${ }^{4}$ and in $60 \%$ of angiomyolipomas from patients with sporadic lymphangioleiomyomatosis. ${ }^{5}$ Somatic TSC2 mutations and loss of heterozygosity have been found in both angiomyolipomas and microdissected pulmonary lymphangioleiomyomatosis cells from patients with the sporadic form of lymphangioleiomyomatosis, supporting the twohit model for lymphangioleiomyomatosis pathogenesis. ${ }^{6,7}$ These studies suggest that sporadic lymphangioleiomyomatosis and tuberous sclerosis complex have a common genetic basis and implicate the Rheb/mammalian target of Rapamycin (mTOR)/p70 S6 kinase (p70 S6K) signaling pathway in the pathogenesis of angiomyolipomas and lymphangioleiomyomatosis.

The mTOR signaling pathway integrates cues from the cell cycle, energy status, and growth factors to control cell size and proliferation. Activation of mTOR results in the phosphorylation of p70 S6K and 4E-BP1 and the downstream phosphorylation of ribosomal protein S6 (S6) resulting in translational initiation and cell growth. Ras homolog-enriched in brain (Rheb), a member of the Ras family of oncogenes, has been shown to directly activate mTOR. ${ }^{8}$ Rheb, like other Ras family members, cycles between an active GTP-bound and an inactive GDPbound state. The hamartin/tuberin complex negatively regulates Rheb by converting Rheb-GTP to Rheb-GDP, thereby inactivating Rheb and inhibiting mTOR. Recently, a protein with $73 \%$ homology (52\% identity) to Rheb was identified and designated Rheb-like protein (RhebL1, also known as Rheb2). Similar to Rheb, overexpression of RhebL1 activates mTOR and induces p70 S6K and S6 phosphorylation. . $^{9,10}$

It is known that mTOR activation is present in $60 \%$ of lymphangioleiomyomatosis-associated angiomyolipomas with TSC2 loss of heterozygosity. ${ }^{11}$ However, it is not known whether mTOR is activated in lymphangioleiomyomatosis-associated angiomyolipomas lacking TSC1/ TSC2 loss of heterozygosity. In this study, we found moderate or strong phospho-S6 (Ser235/236) immunoreactivity in all lymphangioleiomyomatosis-associated and sporadic angiomyolipomas examined (100\%), suggesting a high incidence of mTOR/p70 S6K signaling pathway activation in these tumors.

\section{Materials and methods}

\section{Tissue}

This study was approved by the Institutional Review Board of Fox Chase Cancer Center. Paraffinembedded tissue sections, $4 \mu \mathrm{m}$, were used for immunohistochemical analysis; $10 \mu \mathrm{m}$ sections were used for DNA extraction.

\section{Immunohistochemistry}

Paraffin sections were deparaffinized in xylene and rehydrated in a graded series of ethanol. For antigen retrieval, sections were boiled in Citric Buffer $(10 \mathrm{mM}$ sodium citrate-trisodium salt dihydrate, Sigma, St Louis, MO, USA), pH 6.0, for $10 \mathrm{~min}$. Endogenous peroxidase activity was blocked with $3 \%$ hydrogen peroxide in methanol for $30 \mathrm{~min}$ at room temperature. Nonspecific background was eliminated by incubating the tissue with nonimmune serum (Histostain-Plus kit, Zymed, San Francisco, CA, USA) for $30 \mathrm{~min}$ at room temperature. The sections were then incubated in a humidified chamber with rabbit polyclonal antibodies against phospho-S6 ribosomal protein (Ser235/236, Cell Signaling Technology, Beverly, MA, USA) or tuberin (C-20, Santa Cruz Biotechnology, Santa Cruz, CA, USA) overnight at $4^{\circ} \mathrm{C}$. The slides were developed using the Histostain-Plus kit (Zymed) according to the manufacturer's instructions and counterstained with hematoxylin. Negative controls included omission of the primary antibody and substitution of rabbit immunoglobulin IgG (Santa Cruz Biotechnology, Santa Cruz, CA, USA) for the primary antibody.

Two independent observers scored the slides semiquantitatively: negative $(-)$ indicated no staining, one plus $(+)$ indicated weakly positive staining, two plus $(++)$ indicated moderately positive staining, and three plus $(+++)$ indicated strongly positive staining. Slides containing tissue with regions of variable staining were scored for both degrees of intensities (eg, $++/+++$ ).

\section{Rheb and RhebL1 Mutational Analysis}

DNA was extracted from paraffin sections as described previously. ${ }^{12}$ Briefly, paraffin sections were scraped into $300 \mu \mathrm{l}$ Histology Slide Buffer (50 mM, $10 \mathrm{mM}$ Tris, pH8.3, $1.5 \mathrm{mM} \mathrm{MgCl}_{2}, 100 \mu \mathrm{g} /$ ml BSA, $0.45 \%$ Tween 20, 0.45\% NP40) plus $20 \mu \mathrm{g} /$ $\mathrm{ml}$ Proteinase K (Sigma) and incubated at $65^{\circ} \mathrm{C}$ overnight. Samples were incubated at $95^{\circ} \mathrm{C}$ for $10 \mathrm{~min}$ to inactivate Proteinase $\mathrm{K}$ and centrifuged at 14000 r.p.m. for $10 \mathrm{~min}$. Supernatant was diluted 1:10 in distilled water and $5 \mu \mathrm{l}$ were used in PCR 
reactions. Intronic oligonucleotide primers were generated based on the human Rheb (Genebank Accession: BC066307) and RhebL1 (BC027482) cDNA sequence and designed to span exons 3 or 4 of Rheb and RhebL1. The following sets of primer sequences were used: RhebL1-exon 3F: 5'-CAG GGG ACA TAC ATC GCT-3' ${ }^{\prime}$ and RhebL1-exon 3R: 5'-GGA TGA GCC ACA AAG ACT-3'; RhebL1-exon 4F: 5'GCT GTT CCA TAA ATA CAT- $3^{\prime}$ and RhebL1-exon 4R: 5'-AAC TTT ATG AGA CAG TCC-3'; Rheb-exon 3F: 5'-CGA GTT AAT TTT TTT AAA CTA ATG-3' and Rheb-exon 3R: $5^{\prime}$-GCT GTA TAA GAT TCT GAG ATA CC-3'; Rheb-exon 4F: 5'-TGT GAT GCT ATA TGA AGA CTA CT-3' and Rheb-exon 4R: $3^{\prime}$-AAG GAT CAA TGT TAT CTA TGA GTA-3'. Diluted DNA ( $5 \mu \mathrm{l}$ ) was added to a $25 \mu \mathrm{l}$ PCR reaction containing $12.5 \mu \mathrm{l}$ of JumpStart Taq ReadyMix (Sigma) and $10 \mu \mathrm{M}$ each of the forward and reverse primers. PCR reactions were amplified in a PTC-200 thermocycler (Bio-Rad Laboratories, Waltham, MA, USA) for 35 cycles with an annealing temperature of $53^{\circ} \mathrm{C}$ (for RhebL1) or $56^{\circ} \mathrm{C}$ (for Rheb). PCR products were purified using the QIAquik PCR purification kit (Qiagen, Valencia, CA, USA) and sequenced in both the forward and reverse directions.

\section{Results}

Immunohistochemistry on

Lymphangioleiomyomatosis-Associated

Angiomyolipomas

To determine whether the mTOR/p70 S6K pathway is activated in sporadic lymphangioleiomyomatosisassociated angiomyolipomas lacking TSC1/TSC2 loss of heterozygosity we focused on five tumors in which loss of heterozygosity in the TSC1 region of chromosome 9q34 and the TSC2 region of chromosome 16p13 (Table 1) was previously excluded. ${ }^{5}$ DNA was extracted from both angiomyolipoma and normal tissue from the same individual and used in PCR reactions using markers on chromosome 9q34 for TSC1 or markers on chromosome 16p13 for TSC2. Loss of heterozygosity was determined by comparing the allele pattern of the microsatellite markers from angiomyolipoma DNA to the two copy allele pattern in normal kidney DNA from the same individual. Retinal, dermatological, and neurological assessments of these patients yielded no clinical evidence of tuberous sclerosis complex. ${ }^{5}$ Two of these tumors (\#489 and \#491) were also previously analyzed for mutations in all 41 exons of TSC2 using single-strand conformation polymorphism analysis, in which exons with aberrant banding patterns are directly sequenced to identify variations. ${ }^{6}$ Tumor 491 had a 4-bp deletion in exon 5 (nucleotides 529532) that is predicted to cause premature protein truncation; a 'second hit mutation' was not identified in this tumor. No TSC2 mutations were found in tumor 489.

Immunohistochemical staining of these tumors using a polyclonal antibody targeted against the C-terminus of tuberin was used to confirm the presence of full-length protein. Strongly positive $(+++)$ tuberin staining was observed in the tubular epithelium of a normal kidney specimen (Figure 1a) and in normal kidney tissue adjacent to angiomyolipomas (not shown). Moderate $(++)$ or strong $(+++)$ tuberin immunoreactivity was evident in all sporadic lymphangioleiomyomatosis-associated

Table 1 Summary of immunohistochemical staining and mutational analysis of angiomyolipomas

\begin{tabular}{|c|c|c|c|c|c|c|c|c|}
\hline \multirow[t]{2}{*}{ Case \# } & \multirow[t]{2}{*}{$T S C 1 L O H^{a}$} & \multirow[t]{2}{*}{ TSC2 $L O H^{\mathrm{a}}$} & \multirow[t]{2}{*}{ Tuberin staining } & \multirow[t]{2}{*}{ Phospho-S6 staining } & \multicolumn{2}{|c|}{$R h e b^{\mathrm{c}}$} & \multicolumn{2}{|c|}{ RhebL $^{\mathrm{c}}$} \\
\hline & & & & & Exon 3 & Exon 4 & Exon 3 & Exon 4 \\
\hline \multicolumn{9}{|c|}{ Lymphangioleiomyomatosis-associated angiomyolipomas } \\
\hline 443 & - & - & $+/++$ & $++/+++$ & wt & wt & wt & wt \\
\hline 480 & - & - & ++ & ++ & $\ldots$ & $\ldots$ & wt & wt \\
\hline 481 & - & - & +++ & +++ & wt & wt & wt & wt \\
\hline 489 & - & - & $+/++$ & +++ & wt & wt & wt & wt \\
\hline 491 & - & - & ++ & +++ & wt & wt & wt & wt \\
\hline \multicolumn{9}{|c|}{ Sporadic angiomyolipomas } \\
\hline 1 & - & - & $\ldots$ & $\ldots$ & wt & wt & wt & wt \\
\hline 4 & - & - & $\ldots$ & $\ldots$ & wt & wt & wt & wt \\
\hline 20 & - & - & $\ldots$ & $\ldots$ & wt & wt & wt & wt \\
\hline 24 & - & - & $\ldots$ & $\ldots$ & wt & wt & wt & wt \\
\hline 31 & - & - & +++ & ++ & wt & wt & wt & wt \\
\hline 40 & - & - & $++/+++$ & +++ & wt & wt & wt & wt \\
\hline 58 & - & - & +++ & +++ & wt & wt & wt & wt \\
\hline 110 & - & - & $\ldots$ & $\ldots$ & wt & wt & wt & wt \\
\hline
\end{tabular}

${ }^{\mathrm{a}}$ TSC1 and TSC2 loss of heterozygosity (LOH) status was previously determined: a minus sign (-) indicates absence of LOH. ${ }^{4,5}$

${ }^{\mathrm{b}}$ Staining was graded semiquantitatively as outlined in Materials and methods.

${ }^{\mathrm{c}}$ Rheb and RhebL1 mutational analysis was performed as outlined in Materials and methods: wt indicates that the exon was wild type. An ellipsis (...) indicates data not determined. 

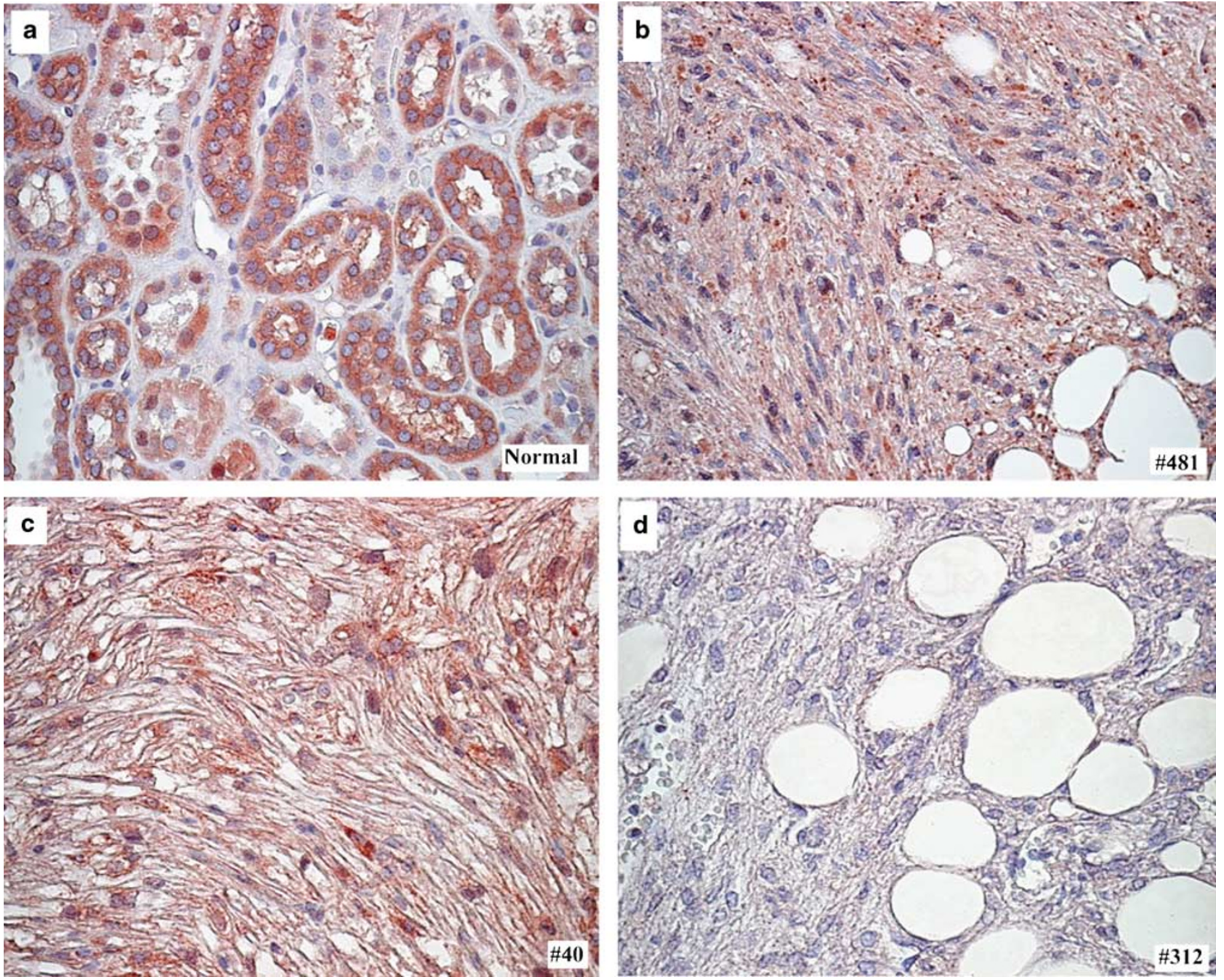

Figure 1 Tuberin expression in angiomyolipomas. Tuberin expression in normal kidney (a) compared to a lymphangioleiomyomatosisassociated (b) and sporadic (c) angiomyolipoma. Numbers in lower right corners refer to case numbers. An angiomyolipoma from a tuberous sclerosis complex patient with a germline TSC2 mutation showed a loss of tuberin expression $(\mathbf{d})$. Magnification $=400 \times$

angiomyolipomas (Figure 1b, summarized in Table 1). An angiomyolipoma from a tuberous sclerosis complex patient with a germline TSC2 mutation was included as a control and demonstrated weak tuberin staining, as expected (Figure 1d).

The phosphorylation of ribosomal protein S6 is widely used as an indicator of mTOR activity in human tumor specimens, and has been shown to be strongly positive in tumor cells derived from patients with tuberous sclerosis complex. ${ }^{11,13} \mathrm{Im}$ munohistochemical staining of these angiomyolipomas was performed to determine whether there is an increase in phosphorylated S6 despite the presence of tuberin. Weakly positive focal phospho-S6 staining was observed in the tubular epithelium of normal kidney (Figure 2a) and in normal kidney tissue adjacent to angiomyolipomas. In contrast, moderate or strong phospho-S6 (Ser235/236) immunoreactivity was evident in all sporadic lymphangioleiomyomatosis-associated angiomyoli- pomas (100\% positive, Table 1 and Figure 2), suggesting a high incidence of mTOR/p70 S6 K signaling pathway activation in these tumors. Strong phospho-S6 immunoreactivity was present in all three characteristic components of angiomyolipomas: smooth muscle cells (Figure 2b), adipose cells (Figure 2c) and cells surrounding dysplastic blood vessels (Figure 2d). As a positive control for phospho-S6 staining, a TSC-associated angiomyolipoma was included and demonstrated strong phospho-S6 staining, as expected (not shown).

\section{Immunohistochemistry on Pulmonary Lymphangioleiomyomatosis}

Lymphangioleiomyomatosis pulmonary samples from patients \#489 and \#491 were also analyzed for phospho-S6 immunoreactivity. Similar to the angiomyolipomas, positive phospho-S6 (Ser235/ 

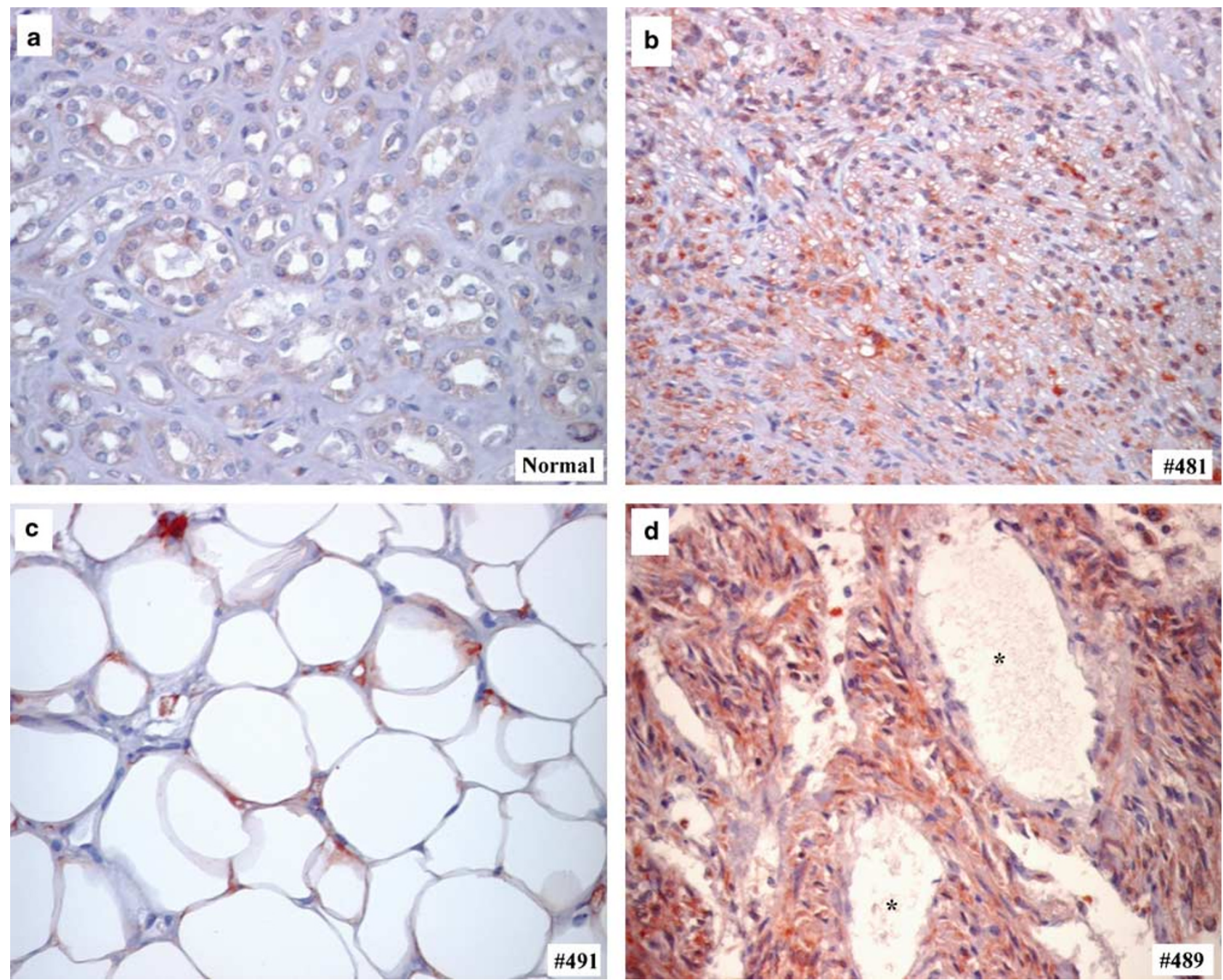

Figure 2 Hyperphosphorylation of S6 (Ser235/236) in lymphangioleiomyomatosis-associated angiomyolipomas. Phospho-S6 staining of normal kidney (a) compared to three tumors representing characteristic components of angiomyolipomas (b, c, and d). Strong phosphoS6 ribosomal protein immunoreactivity is illustrated in the smooth muscle component of tumor 481 (b), in the fat component of tumor 491 (c), and in cells in the vascular component of tumor 489 (d, asterisks denote the vessel lumens). Numbers in lower right corners refer to case numbers. Magnification $=400 \times$.
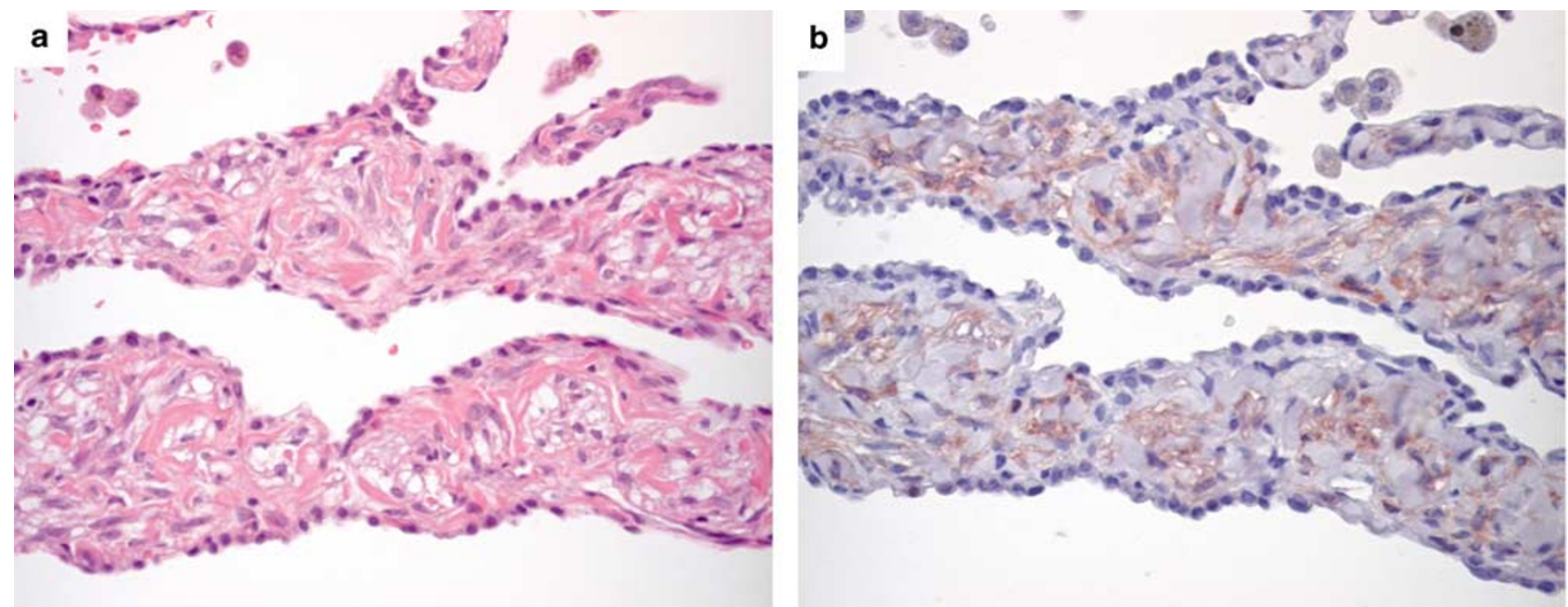

Figure 3 Hyperphosphorylation of S6 in pulmonary lymphangioleiomyomatosis. H\&E (a) and phospho-S6 (b) staining of pulmonary tissue from patient \#489. Focal phospho-S6 staining is present in pulmonary lymphangioleiomyomatosis cells. Magnification $=400 \times$. 
236) staining was evident in both lymphangioleiomyomatosis pulmonary samples (Figure 3 ), although the pattern was strikingly focal (ie, not all clusters of lymphangioleiomyomatosis cells were immunoreactive). Adjacent lung tissue was negative for phospho-S6 (not shown).

\section{Immunohistochemistry on Sporadic Angiomyolipomas Independent of Lymphangioleiomyomatosis}

We also tested three sporadic angiomyolipomas from patients who were not diagnosed with either tuberous sclerosis complex or lymphangioleiomyomatosis. These angiomyolipomas were previously shown to lack TSC1/TSC2 loss of heterozygosity $^{4}$ and retention of tuberin expression was confirmed in these tumors (Figure 1c, Table 1). Similar to lymphangioleiomyomatosis-associated angiomyolipomas, moderate or strong phospho-S6 (Ser235/236) immunoreactivity was evident in all sporadic angiomyolipomas analyzed (summarized in Table 1).

\section{Mutational Analysis of Rheb and RhebL1}

As the phospho-S6 immunohistochemistry indicated mTOR activation without a loss of tuberin, we next asked whether the mTOR/p70 S6K pathway activation in these sporadic and lymphangioleiomyomatosis-associated angiomyolipomas was the result of mutational activation of Rheb and/or RhebL1, direct activators of mTOR. Gln61 in Ras, the most frequently mutated residue in human cancer, ${ }^{14}$ is homologous to Gln64 in both Rheb and RhebL1. For both Rheb and RhebL1, Gln64 is the last codon before the intron that separates exons 3 and 4 . Therefore, activating mutations of Rheb and RhebL1 were sought by direct sequencing of exons 3 and 4 of $R h e b$ and RhebL1 from these tumors. Five additional sporadic angiomyolipomas which lack TSC1/TSC2 loss of heterozygosity ${ }^{4}$ were also included in the mutational analysis. Limited tissue availability for these angiomyolipomas precluded their use for phospho-S6 immunohistochemistry. As the fixatives used in archival tissue are damaging to nucleic acids, it is difficult to amplify targets larger than 300 bases. ${ }^{12}$ As such, intronic primers were designed to specifically amplify either exon 3 or 4 of Rheb and RhebL1 from archival tissue-extracted DNA. The amplification and sequencing of exons 3 and 4 of RhebL1 was successful for all of the 13 angiomyolipomas examined, and successful results for $R$ heb were obtained in 12 of the 13 cases (summarized in Table 1). No mutations in exons 3 and 4 of either Rheb or RhebL1 were identified, suggesting that mutational activation of Rheb or RhebL1 is not a frequent mechanism of activation in lymphangioleiomyomatosis-associated and sporadic angiomyolipomas.

\section{Discussion}

Activation of the mTOR pathway has been previously demonstrated in TSC-associated ${ }^{13}$ and sporadic $^{11}$ angiomyolipomas, and it is known that some cases of sporadic lymphangioleiomyomatosis are associated with somatic TSC2 mutation and loss of heterozygosity in both angiomyolipoma and pulmonary lymphangioleiomyomatosis cells. ${ }^{6,7}$ This study was focused specifically on the status of mTOR activation in sporadic lymphangioleiomyomatosis angiomyolipomas lacking TSC1/TSC2 loss of heterozygosity. We found that all lymphangioleiomyomatosis-associated angiomyolipomas examined demonstrated strong phospho-S6 immunoreactivity, suggesting a high incidence of mTOR/p70 S6K signaling pathway activation despite the lack of TSC1/TSC2 loss of heterozygosity. Sporadic angiomyolipomas from patients not known to have tuberous sclerosis complex or lymphangioleiomyomatosis also demonstrated moderate or strong phospho-S6 immunoreactivity. This is the first report of $\mathrm{mTOR} / \mathrm{p} 70$ S6K pathway activation in sporadic and lymphangioleiomyomatosis-associated angiomyolipomas lacking TSC1/TSC2 loss of heterozygosity.

Rheb and RhebL1 directly activate mTOR. ${ }^{8}$ Therefore, we hypothesized that mutational activation of Rheb and/or RhebL1 could be the underlying cause of mTOR activation in angiomyolipomas lacking TSC1/TSC2 loss of heterozygosity. We focused on exons 3 and 4 of Rheb and RhebL1 since Gln61 in Ras is the most frequently mutated residue in human cancer ${ }^{14}$ and is homologous to Gln64 in both Rheb and RhebL1, the last codon before the intron that separates exons 3 and 4 . We did not detect $R h e b$ or RhebL1 mutations in five lymphangioleiomyomatosis-associated angiomyolipomas or eight sporadic angiomyolipomas.

Our mutational analysis does not preclude the possibility that other activating mutations are present in Rheb or RhebL1 outside of exons 3 and 4, although this is unlikely. Rheb and RhebL1 are homologs of Ras, and Ras mutations have been previously reported at only three codons: Gly12, Gln61, and more rarely Gly13. ${ }^{15,16}$ Ras-proteins with these somatic mutations have defective GTPase activity resulting in constitutive activation and, consequently, sustained cell proliferation and tumor formation. Mutation of Ras Gly12 to a number of other residues, including arginine, results in constitutive activation. ${ }^{15,17}$ However, both Rheb and RhebL1 already contain an arginine (Arg15) at the position equivalent to Gly12 in Ras, suggesting that Rheb and RhebL1 are already in the activated state and possibly accounts for the higher intrinsic activity of Rheb compared with Ras. ${ }^{18}$ Another possible mechanism for mTOR activation in these tumors is chromosomal amplification of Rheb or RhebL1. The Rheb gene is located on chromosome $7 q 36^{19}$ and trisomy 7 has been reported in renal 
angiomyolipomas. However, the physiological relevance of this finding is controversial since trisomy 7 has also been observed in normal kidney cells. ${ }^{20,21}$ It is also possible that these angiomyolipomas have somatic mutations inactivating both alleles of TSC1 or TSC2 that would not be detected in a loss of heterozygosity analysis. Immunohistochemical analysis of these tumors using an antibody targeted to the C-terminus of tuberin indicated the presence of full-length protein, although it is still possible that undetected TSC2 inactivating mutations resulting in small in-frame insertions/deletions or missense amino-acid changes may be present. Alternatively, constitutive stimulation of upstream signaling pathways may account for mTOR activation in these tumors. For example, tuberin is phosphorylated and inactivated by Erk $2^{22}$ and $\mathrm{Akt}^{23}$ and activation of either of these pathways is predicted to result in Rheb/mTOR/p70 S6K pathway activation.

Similar to the angiomyolipomas, positive phospho-S6 staining was evident in two matched lymphangioleiomyomatosis pulmonary samples, although not all lymphangioleiomyomatosis cells were immunoreactive. It is possible that this focal pattern is due to the presence of additional acquired mutations or soluble local factors that repress the mTOR pathway in the lung. A previous report examined phospho-S6 immunoreactivity in three cases of pulmonary lymphangioleiomyomatosis and found that most lymphangioleiomyomatosis cells were phospho-S6 positive, ${ }^{24}$ suggesting that our staining pattern may be unique to these two samples and/or the antibody used. The smooth muscle cells of pulmonary lymphangioleiomyomatosis and angiomyolipomas appear to be closely related histologically and immunohistochemically ${ }^{5,25}$ and, in some cases, contain identical TSC2 mutations or loss of heterozygosity ${ }^{7,26}$ suggesting a model in which renal angiomyolipoma cells migrate or metastasize to the lung resulting in pulmonary lymphangioleiomyomatosis. ${ }^{26}$ Evidence of mTOR activation in both angiomyolipomas and pulmonary lymphangioleiomyomatosis in this study lends further credence to this model. Thus, identifying the mechanisms underlying the pathogenesis of angiomyolipomas is likely to contribute towards the development of successful targeted therapies for lymphangioleiomyomatosis patients. Further work is needed to identify the mechanism of mTOR activation independent of TSC1/TSC2 loss of heterozygosity as well as determine the potential benefits of pharmaceutical targeting of the mTOR pathway for this subset of lymphangioleiomyomatosis patients.

In summary, we found that $100 \%$ of angiomyolipomas, regardless of disease background, have hyperphosphorylation of the mTOR/p70 S6K signaling pathway. Activating mutations were not found in predicted 'hotspots' of either Rheb or RhebL1, suggesting that activation of the mTOR/p70 S6K signaling pathway in angiomyolipomas may occur through mechanisms other than Rheb/RhebL1 mutational activation. Identifying the mechanism of this activation could lead to novel therapeutic concepts for patients with lymphangioleiomyomatosis. Moreover, this high incidence of mTOR signaling pathway activation suggests that treatment with Rapamycin and its analogues may benefit patients with angiomyolipomas independent of the detection of loss of heterozygosity in the TSC1 or TSC2 gene regions.

\section{Acknowledgements}

We gratefully acknowledge the Fox Chase Cancer Center DNA Sequencing Facility for their assistance. This work was supported by National Institutes of Health Grant HL 60746.

\section{References}

1 Castro M, Shepherd CW, Gomez MR, et al. Pulmonary tuberous sclerosis. Chest 1995;107:189-195.

2 Eble JN. Angiomyolipoma of kidney. Semin Diagn Pathol 1998;15:21-40.

3 Henske EP, Scheithauer BW, Short MP, et al. Allelic loss is frequent in tuberous sclerosis kidney lesions but rare in brain lesions. Am J Hum Genet 1996;59: 400-406.

4 Henske EP, Neumann HP, Scheithauer BW, et al. Loss of heterozygosity in the tuberous sclerosis (TSC2) region of chromosome band 16p13 occurs in sporadic as well as TSC-associated renal angiomyolipomas. Genes Chromosomes Cancer 1995;13:295-298.

5 Smolarek TA, Wessner LL, McCormack FX, et al. Evidence that lymphangiomyomatosis is caused by TSC2 mutations: chromosome 16p13 loss of heterozygosity in angiomyolipomas and lymph nodes from women with lymphangiomyomatosis. Am J Hum Genet 1998;62:810-815.

6 Carsillo T, Astrinidis A, Henske EP. Mutations in the tuberous sclerosis complex gene TSC2 are a cause of sporadic pulmonary lymphangioleiomyomatosis. Proc Natl Acad Sci USA 2000;97:6085-6090.

7 Maruyama H, Seyama K, Sobajima J, et al. Multifocal micronodular pneumocyte hyperplasia and lymphangioleiomyomatosis in tuberous sclerosis with a TSC2 gene. Mod Pathol 2001;14:609-614.

8 Long X, Lin Y, Ortiz-Vega S, et al. Rheb binds and regulates the mTOR kinase. Curr Biol 2005;15:702-713.

9 Tee AR, Blenis J, Proud CG. Analysis of mTOR signaling by the small G-proteins, Rheb and RhebL1. FEBS Lett 2005;579:4763-4768.

10 Tabancay Jr AP, Gau CL, Machado IM, et al. Identification of dominant negative mutants of Rheb GTPase and their use to implicate the involvement of human Rheb in the activation of p70S6K. J Biol Chem 2003;278: 39921-39930.

11 Karbowniczek M, Yu J, Henske EP. Renal angiomyolipomas from patients with sporadic lymphangiomyomatosis contain both neoplastic and non-neoplastic vascular structures. Am J Pathol 2003;162:491-500.

12 Astrinidis A, Henske EP. Mutation detection in tumor suppressor genes using archival tissue specimens. In: Kearns-Jonker M (ed). Congenital Heart Disease: 
Molecular Diagnostics. Humana Press: Totowa, NJ, 2006, pp 185-196.

13 El-Hashemite N, Zhang H, Henske EP, et al. Mutation in TSC2 and activation of mammalian target of rapamycin signalling pathway in renal angiomyolipoma. Lancet 2003;361:1348-1349.

14 Scheffzek K, Ahmadian MR, Wittinghofer A. GTPaseactivating proteins: helping hands to complement an active site. Trends Biochem Sci 1998;23:257-262.

15 Bos JL. ras oncogenes in human cancer: a review. Cancer Res 1989;49:4682-4689.

16 Bos JL, Toksoz D, Marshall CJ, et al. Amino-acid substitutions at codon 13 of the N-ras oncogene in human acute myeloid leukaemia. Nature 1985;315:726-730.

17 Seeburg PH, Colby WW, Capon DJ, et al. Biological properties of human c-Ha-ras1 genes mutated at codon 12. Nature 1984;312:71-75.

$18 \mathrm{Im}$ E, von Lintig FC, Chen J, et al. Rheb is in a high activation state and inhibits B-Raf kinase in mammalian cells. Oncogene 2002;21:6356-6365.

19 Mizuki N, Kimura M, Ohno S, et al. Isolation of cDNA and genomic clones of a human Ras-related GTPbinding protein gene and its chromosomal localization to the long arm of chromosome 7, 7q36. Genomics 1996;34:114-118.

20 Dal Cin P, Sciot R, Van Poppel H, et al. Chromosome analysis in angiomyolipoma. Cancer Genet Cytogenet 1997;99:132-134.
21 Wullich B, Henn W, Siemer S, et al. Clonal chromosome aberrations in three of five sporadic angiomyolipomas of the kidney. Cancer Genet Cytogenet 1997;96:42-45.

22 Ma L, Chen Z, Erdjument-Bromage H, et al. Phosphorylation and functional inactivation of TSC2 by Erk implications for tuberous sclerosis and cancer pathogenesis. Cell 2005;121:179-193.

23 Dan HC, Sun M, Yang L, et al. Phosphatidylinositol 3kinase/Akt pathway regulates tuberous sclerosis tumor suppressor complex by phosphorylation of tuberin. J Biol Chem 2002;277:35364-35370.

24 Goncharova EA, Goncharov DA, Eszterhas A, et al. Tuberin regulates p70 S6 kinase activation and ribosomal protein S6 phosphorylation. A role for the TSC2 tumor suppressor gene in pulmonary lymphangioleiomyomatosis (LAM). J Biol Chem 2002;277: 30958-30967.

25 Chan JK, Tsang WY, Pau MY, et al. Lymphangiomyomatosis and angiomyolipoma: closely related entities characterized by hamartomatous proliferation of HMB45-positive smooth muscle. Histopathology 1993;22: $445-455$

26 Karbowniczek M, Astrinidis A, Balsara BR, et al. Recurrent lymphangiomyomatosis after transplantation: genetic analyses reveal a metastatic mechanism. Am J Respir Crit Care Med 2003;167: 976-982. 